\title{
The SONAR study - is there a future for endothelin receptor antagonists in diabetic kidney disease?
}

\author{
Avivit Cahn', Simona Cernea ${ }^{2,3}$, Itamar $\operatorname{Raz}^{1}$ \\ ${ }^{1}$ The Diabetes Unit, Department of Endocrinology and Metabolism, Hadassah Medical Center, Hebrew Univercity of Jerusalem, The Faculty of \\ Medicine, Jerusalem, Israel; ${ }^{2}$ Department M3/Internal Medicine IV, University of Medicine, Pharmacy, Science and Technology of Târgu Mureş, \\ Romania; ${ }^{3}$ Diabetes, Nutrition and Metabolic Diseases Outpatient Unit, Emergency County Clinical Hospital, Târgu Mureş, Romania \\ Correspondence to: Dr. Avivit Cahn. The Diabetes Unit, Department of Endocrinology and Metabolism Unit Hadassah Hebrew University Hospital, \\ PO Box 12000, Jerusalem 91120, Israel. Email: avivit@hadassah.org.il. \\ Provenance: This is an invited article commissioned by the Academic Editor Dr. Xiaoyun He (Xiangya Hospital of Central South University, \\ Changsha, China). \\ Comment on: Heerspink HJL, Parving HH, Andress DL, et al. Atrasentan and renal events in patients with type 2 diabetes and chronic kidney disease \\ (SONAR): a double-blind, randomised, placebo-controlled trial. Lancet 2019;393:1937-47.
}

Submitted Sep 05, 2019. Accepted for publication Sep 20, 2019.

doi: 10.21037/atm.2019.09.117

View this article at: http://dx.doi.org/10.21037/atm.2019.09.117

Diabetic kidney disease (DKD) is a clinical diagnosis of chronic kidney disease (CKD) in a patient with diabetes mellitus in the absence of signs or symptoms of other primary causes of kidney damage. It results mainly from microvascular and metabolic changes within the kidneys, and has a distinct histopathological pattern (1-3). Patients with type 2 diabetes mellitus (T2DM), often suffer of multiple comorbidities including obesity, hypertension, renovascular disease, recurrent urinary tract infections or drug toxicities, or may have been subjected to episodes of acute kidney injury, all which can lead to CKD independent of diabetes $(2,3)$. Therefore, the causes of CKD in patients with T2DM may be DKD or non-DKD (NDKD), and in fact, the prevalence of DKD in kidney biopsies from patients with T2DM varies quite largely in different studies, with $14-83 \%$ of patients having biopsies compatible with NDKD and many having combined histologic features (3). In spite of advances in the care of patients with diabetes, the prevalence of DKD is steadily increasing and diabetes accounts for approximately half of the cases of end stage renal disease (ESRD) in developed countries (4). It is estimated that half of the patients with T2DM will develop CKD over the course of their lifetime (4).

Multiple interventions to slow the progression of DKD have been studied, with the clinical endpoints including ESRD, renal death, doubling of serum creatinine or persistent decline in eGFR below a specific predefined threshold. Albuminuria is considered as a surrogate marker of renal deterioration, although it is frequently absent in DKD $(4,5)$, and an effect on hard renal endpoints is required in order to establish the benefit of a drug or intervention.

Intensive glucose control has been shown in many large prospective randomized controlled studies to delay the onset and progression of albuminuria in patients with T1DM and T2DM (1,2). A positive effect of intensive glucose control on renal outcomes, assessed by ESRD or creatinine based endpoints, has been shown in the ADVANCE trial, as well as in the extension of the UKPDS and DCCT-EDIC studies, yet the effect is observed after a time lag of several years and is more prominent in those with earlier stage CKD (6). Blood pressure control, regardless of the agents used, has been shown to reduce the risk of albuminuria and of eGFR deterioration $(1,2)$.

For nearly two decades, the only drugs indicated for the treatment of DKD included inhibitors of the reninangiotensin system (RAS), whereby these agents have demonstrated a significant role in reversing albuminuria and slowing eGFR decline $(1,2)$. The novel classes of glucose lowering agents have also been studied for potential renal benefits. DPP-4 inhibitors have a small effect of reduced albuminuria, yet no effect on renal outcomes (7). 
GLP-1 receptor agonists have shown a $17 \%$ reduction in the broad composite kidney outcome [HR (95\% CI) 0.83 $(0.69,1.00), \mathrm{P}=0.047]$, which was driven by a reduction in macroalbuminuria only [HR $(95 \% \mathrm{CI}) 0.76(0.68,0.86)$, $\mathrm{P}<0.003$ ], as indicated by a recent meta-analysis of seven cardiovascular (CV) outcome trials (8). Contrariwise, the recently approved class of glucose lowering agentsthe SGLT2 inhibitors-demonstrated a marked effect on reducing ESRD with reductions of approximately $50 \%$ in the composite renal outcome as well as marked improvements in albuminuria (9-11). These agents have also shown robust reduction in hospitalization for heart failure (HHF), with some also demonstrating a reduction in CV death (9-11). A study dedicated to the assessment of renal outcomes of canagliflozin in patients with T2DM was terminated early, after 2.6 years, having attained superiority in its primary endpoint (ESRD, doubling of serum creatinine or death from renal or CV causes) HR (95\% CI) 0.66 (0.53, 0.81), $\mathrm{P}<0.001$ (12). Based on this study, a new drug application has been submitted to the U.S. FDA for treatment of CKD in T2DM (13). The magnitude of effect and its consistency across different drugs in the class increase the likelihood of SGLT2 inhibitors becoming the mainstay of care for patients with DKD in addition to RAS inhibitors (9-12).

The recently published results of the Study of diabetic Nephropathy with AtRasentan (SONAR) study, which evaluated the renal outcomes of an endothelin receptor antagonist in patients with T2DM and CKD, are thus viewed in the context of the emerging cardio-renal efficacy data of SGLT2 inhibitors (14).

Endothelin A receptor antagonists have been studied as potential agents for slowing progression of CKD. Endothein-1 is a potent vasoconstrictor and is present at higher levels in patients with diabetes $v s$. healthy controls. Hyperglycemia, insulin resistance, obesity and RAS activation lead to an increase in its levels (15). Animal studies have shown that selective blockade of the endothelin A receptor downregulates the fibrotic, inflammatory and proliferative actions of endothelin 1 at the kidney level (15). Activation of the endothelin $\mathrm{B}$ receptor inhibits sodium reabsorption; thus its blockade may lead to fluid retention. However, there appears to be a role for the endothelin A receptor as well in promoting natriuresis, therefore even selective blockade of the endothelin A receptor may lead to fluid retention (15). Endothelin receptor blockers must therefore balance between improvement in DKD as reflected by reduced albuminuria $v s$. increased risk of fluid retention and concomitant heart failure.

A clinical study of avosentan, a selective endothelin A receptor antagonist, in patients with T2DM was terminated prematurely, after median follow-up of 4 months (maximum follow-up 16 months). Although proteinuria was reduced with avosentan, it led to significantly more events of fluid overload and congestive heart failure and a trend to increased mortality was observed (16).

Early clinical studies with atrasentan tested several doses of the drug aiming to identify the ideal dose for balancing albuminuria reduction and fluid retentionboth dose dependent effects (17). The RADAR study (Reducing residual Albuminuria in subject with Diabetes and nephropathy with AtRasentan) was a phase 2B study conducted in parallel in the US and in Japan. The study randomized 211 patients with T2DM and macroalbuminuria who were receiving maximally tolerated doses of ACE inhibitors or ARBs to atrasentan 0.75, $1.25 \mathrm{mg} / \mathrm{d}$ or placebo for 12 weeks (18). Atrasentan led to a significant reduction in albuminuria $(-36.2 \%,-43.9 \%$ and $+2 \%$ with atrasentan $0.75,1.25 \mathrm{mg}$ and placebo respectively), yet events of fluid retention were reported more often in the higher dose compared to placebo. Notably, patients with a history of moderate or severe edema, pulmonary edema, pulmonary hypertension, congestive heart failure or those receiving high dose diuretics were excluded from the study.

The optimal dose of atrasentan for subsequent clinical studies was calculated by evaluating the exposure response relationships of albuminuria and bodyweight analyzing data from the RADAR study. The dose of $0.75 \mathrm{mg} / \mathrm{d}$ was identified as being the optimal dose for renal protection with maximal albuminuria reduction and minimal sodium retention (19), although it should be noted that individualized responses were variable. Data from the RADAR study were additionally analyzed with the "Parameter Response Efficacy" score algorithm to ascertain which patients would be more likely to have a renal benefit with the agent. The algorithm determined that patient with $>30 \%$ albuminuria reduction from baseline are those in whom renal outcomes are expected to improve significantly-being the basis for the enrichment process described below (20).

The SONAR study was designed to ascertain the safety and efficacy of atrasentan in slowing the progression of DKD (14). The study included patients with T2DM, an estimated GFR of $25-75 \mathrm{~mL} / \mathrm{min} / 1 / 73 \mathrm{~m}^{2}$, UACR $\geq 300 \mathrm{mg} / \mathrm{g}$ and $<5,000 \mathrm{mg} / \mathrm{g}$ and $\mathrm{BNP} \leq 200 \mathrm{pg} / \mathrm{mL}$. 
Patients with history of severe peripheral edema, pulmonary hypertension, previous HHF or current constellation of symptoms suggestive of heart failure were excluded from the trial. The primary endpoint of the study was a composite of doubling of serum creatinine (sustained for $\geq 30$ days) or ESRD (eGFR $<15 \mathrm{~mL} / \mathrm{min}$ per $1.73 \mathrm{~m}^{2}$ sustained for $\geq 90$ days, chronic dialysis for $\geq 90$ days, kidney transplantation, or death from kidney failure) $(21,22)$.

The study employed a unique enrichment process aiming to include those individuals who may benefit most from the drug without experiencing significant side effects. Patients included in the study, following an initial runin period of 4-12 weeks during which ACE inhibitors/ ARB's and diuretic treatment were optimized, entered a 6-week enrichment period. During the enrichment period all patients received open label atrasentan. Only patients who did not have significant fluid retention (defined as less than $3 \mathrm{~kg}$ weight gain and BNP levels $<300 \mathrm{pg} / \mathrm{mL}$ ), and who did not have an increase in serum creatinine of more than $0.5 \mathrm{mg} / \mathrm{dL}$ and $20 \%$ from baseline, were subsequently randomized. Moreover, only patients responding to treatment, as defined by $>30 \%$ reduction in albuminuria were included in the study. The final study randomized 2,648 responders to atrasentan or placebo from the 4,117 patients who completed the enrichment period. Among the non-responders, whose UACR decreased by $<30 \%$ but did not experience significant fluid retention during the enrichment period, 1020 were sequentially randomized to atrasentan or placebo and they were studied in a secondary analysis of the trial.

The trial was terminated prematurely, by the sponsor's decision, since the rate of the primary composite outcome was lower than expected and it appeared the trial will need to be longer than originally planned. Surprisingly, in spite of its early closure, the study did achieve its primary endpoint. After median follow up of 2.2 years, the primary endpoint occurred in $79(6.0 \%)$ patients in the atrasentan group and $105(7.9 \%)$ in the placebo group, HR $(95 \%$ CI) $0.65(0.49,0.88), \mathrm{P}=0.0047$. HHF was insignificantly increased with atrasentan vs. placebo $[3.5 \%$ vs. $2.6 \%$ respectively, HR (95\% CI) 1.33 (0.85, 2.07), $\mathrm{P}=0.208]$, and there was a significant increase in fluid retention. The composite cardiorenal endpoint (doubling of serum creatinine, ESRD, CV death, non-fatal MI and non-fatal stroke) was reduced with atrasentan [HR (95\% CI) 0.80 $(0.64,0.99), \mathrm{P}=0.049]$. Among non-responders, the primary endpoint occurred in $73(14.3 \%)$ patients in the atrasentan group and in $87(17 \%)$ in the placebo group, HR (95\% CI)
$0.75(0.55,1.03), \mathrm{P}=0.079$. Fluid retention and heart failure were more common with atrasentan $v s$. placebo in the nonresponders group as well. The effect of atrasentan on the primary outcome was consistent in responders and in nonresponders (interaction $\mathrm{P}$ value 0.41 ).

In spite of its short duration and early termination, many aspects in the trial's design, population and outcomes merit discussion. Some consideration should be given to the amount of clinical data accumulated prior to setting out for a large, long-term, renal outcome study. Phase 2 program of this drug was not extensive, and most data which determined the study design was from the positive, yet relatively small, RADAR study. Challenges in conducting renal outcome studies are appreciated, as notably 93 sites were included in the RADAR study which recruited only 211 patients. Still, an additional trial in the interim using a similar design to the SONAR may have led to similar conclusions with less resources spent.

A noteworthy aspect of this trial is its enrichment design which is the first to be employed in a large-scale renal outcome study. This design aimed to pinpoint those individuals who may truly benefit from the drug without concomitant side effects, yet, it significantly hampered the external validity of the trial. Indeed, only about half the screened patients entered the run-in period and only a quarter were responders. Although this conduct does mimic a real-life setting in which poor responders and those with side effects are withdrawn from the drug, it precludes extrapolating the drug's effect on a broader population. Moreover, the inclusion of BNP both in the enrollment and as a criterion for response is a significant deviation from the standard of care.

Interestingly, even after employing this unique enrichment strategy the responses to the drug in the non-responder group reached borderline significanceindicating that setting an arbitrary threshold for drug response may not always hold true. Moreover, fluid retention was still more frequently observed in those treated with atrasentan, and there was a trend for increased risk of HHF. It is possible that either the parameters and/or the cutoffs used did not capture the interpersonal differences in drug response, or that patient heterogeneity is too large to enable setting any clinically significant cutoff. Additionally, it is possible that the differential drug response cannot be captured at a single point in time. Subtle changes in an individual's volemic status and in albuminuria occur on a daily basis dependent upon salt and water intake, caloric intake and other varying stressors. Setting criteria for 
responders and non-responders based upon several weeks of treatment may not determine the finite individualized response to the drug.

The trial was terminated early, before reaching the 425 events initially planned for. One may speculate that additional considerations in the early termination of the study included the emergence of outstanding renal efficacy data for the SGLT2 inhibitors. These medications not only demonstrated robust reduction in all renal endpoints assessed but also significantly reduced, rather than increased rate of HHF. The robust reduction in HHF observed with SGLT2 inhibitors, increased clinical awareness to the morbidity and mortality burden associated with HF in patients with diabetes. Introducing in this current clinical atmosphere an agent which increases risk of fluid retention, and possibly of HF, even while leading to superior renal outcomes is debatable.

Better understanding of the pathophysiology of DKD may shed further light on the differential renal effects of SGLT2 inhibitors $v s$. endothelin receptor antagonists. Hyperglycemia leads to activation of SGLT2 receptors with resultant reduced sodium concentration in the macula densa, deactivation of the tubuloglomerular feedback, dilation of the afferent renal arteriole and hyperfiltration. Concomitant secretion of renin promotes vasoconstriction of the efferent arteriole. These hemodynamic effects lead to glomerular hyperfiltration and hypertension. Additionally, hyperglycemia leads to hyper-reabsorption of glucose yielding a massive increase in energy consumption of the proximal tubular cells and inducing relative ischemia. The involvement of SGLT2 and renin in these upstream processes may explain why drugs inhibiting these pathways exert profound effects on renal hemodynamics within a short period of time (3). Conversely, the downstream pathophysiological alterations in CKD including fibrosis, mesangial proliferation and damage to the podocytes may vary according to genetic, environmental and etiological causes of DKD—with possible contribution of NDKD. Agents such as endothelin receptor antagonists and others may be more dependent on genetic predilection and the individual renal pathologies and therefore lead to greater heterogeneity of effect.

Agents acting on the endothelin system need not be completely abandoned in the context of DKD. First, we must bear in mind there were persons in whom the efficacysafety balance was positive. Additionally, novel approaches such as the use of ET traps to reduce overall endothelin levels may yield better clinical responses (23). Finally, after the (justified) hype for SGLT2 inhibitors settles, a residual renal risk remains and new pathways for further improving renal outcomes in patients with DKD are still actively sought.

\section{Acknowledgments}

None.

\section{Footnote}

Conflicts of Interest: AC reports grants and personal fees from AstraZeneca and Novo Nordisk and personal fees from Eli Lilly, Medial EarlySign, Abbott, Gefen Medical, Sanofi, Boehringer Ingelheim, Merck Sharp \& Dohme, and GlucoMe. SC received payment for lectures from AstraZeneca, Boehringer Ingelheim, Berlin-Chemie Menarini, Eli Lilly, MSD, Novo Nordisk, Sanofi, Servier Pharma, for clinical trial Steering Committee meetings as National Lead Investigator for DECLARE-TIMI58 from TIMI Study Group, consultant fees for Advisory Board from AstraZeneca and support for travel to meetings from AstraZeneca, Boehringer Ingelheim, Eli Lilly, Sanofi, MSD, Novo Nordisk, Worwag Pharma. IR reports personal fees from AstraZeneca, Bristol-Myers Squibb, Boehringer Ingelheim, Concenter BioPharma and Silkim, Eli Lilly, Merck Sharp \& Dohme, Novo Nordisk, Orgenesis, Pfizer, Sanofi, SmartZyme Innovation, Panaxia, FuturRx, Insuline Medical, Medial EarlySign, CameraEyes, Exscopia, Dermal Biomics, Johnson \& Johnson, Novartis, Teva, GlucoMe, and DarioHealth.

Etbical Statement: The authors are accountable for all aspects of the work in ensuring that questions related to the accuracy or integrity of any part of the work are appropriately investigated and resolved.

\section{References}

1. American Diabetes Association. Microvascular Complications and Foot Care: Standards of Medical Care in Diabetes-2019. Diabetes Care 2019;42:S124-38.

2. Umanath K, Lewis JB. Update on Diabetic Nephropathy: Core Curriculum 2018. Am J Kidney Dis 2018;71:884-95.

3. Anders HJ, Huber TB, Isermann B, Schiffer M. CKD in diabetes: diabetic kidney disease versus nondiabetic kidney disease. Nat Rev Nephrol 2018;14:361-77.

4. Koye DN, Magliano DJ, Nelson RG, Pavkov ME. The 
Global Epidemiology of Diabetes and Kidney Disease. Adv Chronic Kidney Dis 2018;25:121-32.

5. Heerspink HJL, Greene T, Tighiouart H, et al. Chronic Kidney Disease Epidemiology Collaboration. Change in albuminuria as a surrogate endpoint for progression of kidney disease: a meta-analysis of treatment effects in randomised clinical trials. Lancet Diabetes Endocrinol 2019;7:128-39.

6. Wong MG, Perkovic V, Chalmers J, et al. ADVANCEON Collaborative Group. Long-term Benefits of Intensive Glucose Control for Preventing EndStage Kidney Disease: ADVANCE-ON. Diabetes Care 2016;39:694-700.

7. Giugliano D, De Nicola L, Maiorino MI, et al. Type 2 diabetes and the kidney: Insights from cardiovascular outcome trials. Diabetes Obes Metab 2019;21:1790-800.

8. Giugliano D, Maiorino MI, Bellastella G, et al. GLP-1 receptor agonists for prevention of cardiorenal outcomes in type 2 diabetes: an updated meta-analysis including the REWIND and PIONEER 6 trials. Diabetes Obes Metab 2019. [Epub ahead of print].

9. Perkovic V, de Zeeuw D, Mahaffey KW, et al. Canagliflozin and renal outcomes in type 2 diabetes: results from the CANVAS Program randomised clinical trials. Lancet Diabetes Endocrinol 2018;6:691-704.

10. Mosenzon O, Wiviott SD, Cahn A, et al. Effects of dapagliflozin on development and progression of kidney disease in patients with type 2 diabetes: an analysis from the DECLARE-TIMI 58 randomised trial. Lancet Diabetes Endocrinol 2019;7:606-17.

11. Wanner C, Inzucchi SE, Lachin JM, et al. EMPA-REG OUTCOME Investigators. Empagliflozin and Progression of Kidney Disease in Type 2 Diabetes. N Engl J Med 2016;375:323-34.

12. Perkovic V, Jardine MJ, Neal B, et al. CREDENCE Trial Investigators. Canagliflozin and Renal Outcomes in Type 2 Diabetes and Nephropathy. N Engl J Med 2019;380:2295-306.

13. Available online: https://www.jnj.com/janssen-submitssupplemental-new-drug-application-to-u-s-fda-for-

Cite this article as: Cahn A, Cernea S, Raz I. The SONAR study - is there a future for endothelin receptor antagonists in diabetic kidney disease? Ann Transl Med 2019;7(Suppl 8):S330. doi: 10.21037/atm.2019.09.117 invokana-canagliflozin-for-the-treatment-of-chronic-

kidney-disease-in-patients-with-type-2-diabetes

14. Heerspink HJL, Parving HH, Andress DL, et al. SONAR Committees and Investigators. Atrasentan and renal events in patients with type 2 diabetes and chronic kidney disease (SONAR): a double-blind, randomised, placebo-controlled trial. Lancet 2019;393:1937-47.

15. Georgianos PI, Agarwal R. Endothelin A receptor antagonists in diabetic kidney disease. Curr Opin Nephrol Hypertens 2017;26:338-44.

16. Mann JF, Green D, Jamerson K, et al. ASCEND Study Group. Avosentan for overt diabetic nephropathy. J Am Soc Nephrol 2010;21:527-35.

17. Kohan DE, Pritchett Y, Molitch M, et al. Addition of atrasentan to renin-angiotensin system blockade reduces albuminuria in diabetic nephropathy. J Am Soc Nephrol 2011;22:763-72.

18. de Zeeuw D, Coll B, Andress D, et al. The endothelin antagonist atrasentan lowers residual albuminuria in patients with type 2 diabetic nephropathy. J Am Soc Nephrol 2014;25:1083-93.

19. Koomen JV, Stevens J, Mostafa NM, et al. Determining the optimal dose of atrasentan by evaluating the exposureresponse relationships of albuminuria and bodyweight. Diabetes Obes Metab 2018;20:2019-22.

20. Schievink B, de Zeeuw D, Smink PA, et al. Prediction of the effect of atrasentan on renal and heart failure outcomes based on short-term changes in multiple risk markers. Eur J Prev Cardiol 2016;23:758-68.

21. Heerspink HJL, Andress DL, Bakris G, et al. Rationale and protocol of the Study Of diabetic Nephropathy with AtRasentan (SONAR) trial: A clinical trial design novel to diabetic nephropathy. Diabetes Obes Metab 2018;20:1369-76.

22. Heerspink HJL, Andress DL, Bakris G, et al. Baseline characteristics and enrichment results from the SONAR trial. Diabetes Obes Metab 2018;20:1829-35.

23. Jain A, Coffey C, Mehrotra V, et al. Endothelin-1 traps as a potential therapeutic tool: from diabetes to beyond? Drug Discov Today 2019;24:1937-42. 